\title{
Program Design for the dynamic detection of servo system based on Keil Mu Vision3
}

\author{
Wei Youguo ${ }^{1}$, Zhang Xuezhi ${ }^{1}$, Tang Xiaokang ${ }^{1}$, Xiecun $^{1}$, Ma Yongshan $^{2}$ \\ (1.Wuhan Mechanical Technology Colledge, Wuhan, China 2.The 65370 \\ Department,Changchun,China)
}

\begin{abstract}
Key words: servo system; dynamic detection; data acquisition; system simulation
\end{abstract}
\begin{abstract}
As the device in general inspection maintenance, can only carry on the system static parameter measurement, and dynamic performance of the system only can be judged by qualitative observation. In this paper, we adopt modular design scheme and the method based on synchronous sampling and real-time data processing, through online data collection, fitness, amplification, filter and analysis of the dynamic signal, the on-line detection of equipment servo system dynamic parameters is realized. This method can detect the device modulates stationary target speed, the maximum speed, the precision of the tracking error and other dynamic parameters. Therefore, the system fault can be diagnosed to facilitate the rapid maintenance of equipment.

The servo control system is a typical mechanical and electrical control system. Its performance, especially the dynamic performance directly affects the efficiency of the equipment. The device in general inspection maintenance, can only carry on the system static parameter measurement, and some typical fault can be judged. There is in short of dynamic testing means and methods for dynamic performance of the system, and only can be judged by qualitative observation. In this paper, we adopt modular design scheme and the method based on synchronous sampling and real-time data processing, through online data collection, fitness, amplification, filter and analysis of the dynamic signal, the on-line detection of equipment servo system dynamic parameters is realized.
\end{abstract}

\section{1 program design for the dynamic detection system}

\section{1 program design based on Keil $\mu$ Vision3}

As the source program code written in C language cannot be used directly, it is necessary to compile the program and finally generate the . hex file. So we should firstly edit, compile, and debug the program source code by using Keil software, and get the resulting object code in, and then load the .hex file into the Proteus environment of single chip microcomputer to make virtual simulation.

Keil u Vision3 can edit and compile the assembly language and the C51 language, and can connect the target files and the library files, and can create a". hex" file and debug the target program. Thus, users often use Keil $\mathrm{u}$ Vision3 to develop projects to debug process and the resulting ".hex" files are executable code. It is usually used for the development and simulation of single-chip microcomputer system in Proteus.

\subsection{The development process of Keil $\boldsymbol{\mu V}$ Vision 3}

The development process of Keil is just lake the development process of other softwares. That is

(1) create a project, select the target device from the device library, Set the configuration tool;

(2)create the source code using C51 or assembly language program;

(3) generate the application file by the project manager;

(4) modify the errors in the source program;

(5) test the connection.

\section{3 data acquisition and processing}

(1) design the main program and the display function

The designed specific software structure is mainly includes a main program and a display 
function. The main program uses the query mode to query P3.2 mouth of MCU, if it is the low level, that is busy as the $A / D$ conversion is not yet finished, then the main program continues to query until the mouth shows the idle state and it is the high level. The core part of the program is A/D conversion. The designed A/D conversion stop signal uses query mode, and send the end conversion signal through three states door to some bit of the microcontroller's I/O interface. The flow chart of query method is shown in figure 1 .

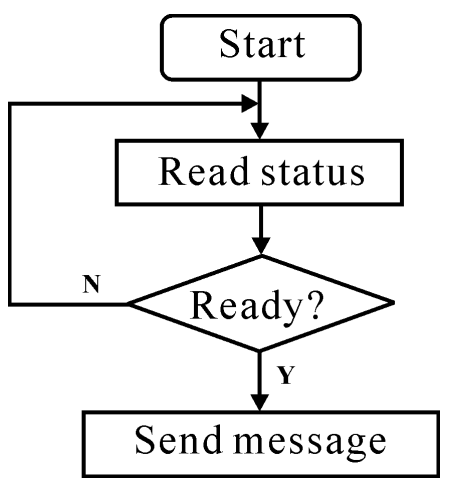

Fig.1 The query transport flow chart

(2) source programming

Connect P0.0, P0.1 and P0.2 of MCU with ADDA, ADDB and ADDC in ADC0808 by the 74LS373 address latch. choose the IN0 acquisition of channels ADC0808. the ADC0808 address is supplied by P0 and P2 mouth. P0 mouth is an address/data multiplex and it provides the lower 8-bit address. The P2 mouth provides the higher 8-bit address. As the voltage derivative from the variable resistor is from IN0 to ADC0808 mouth, so the initial status of the require P0.0,P0.1 and P0.2 is under low level. And as P2.7 controls the ALE and OE ports of ADC0808, so the requirement initial state is low level, therefore the address of ADC0808 can locate 0x7ff8.

\section{Analysis of the system simulation}

\subsection{Simulation for the Data acquisition module}

The simulation implementation steps of data acquisition module are as follows:

(1) Enter the schematic circuit diagram in to the Proteus ISIS;

(2) Edit the C program using the Keil u Vision3, and "hex" documents will be generated after debugging error-free.;

(3) Select add source files in the source menu bar, add program to the target processor;

(4) Set the clock, input, Vcc and other necessary signals and ensure grounding;

(5) Start simulating by selecting start debugging in the debug menu bar;

(6) Solve the emerging problems.

The effect through system simulation is shown in figure 2. 


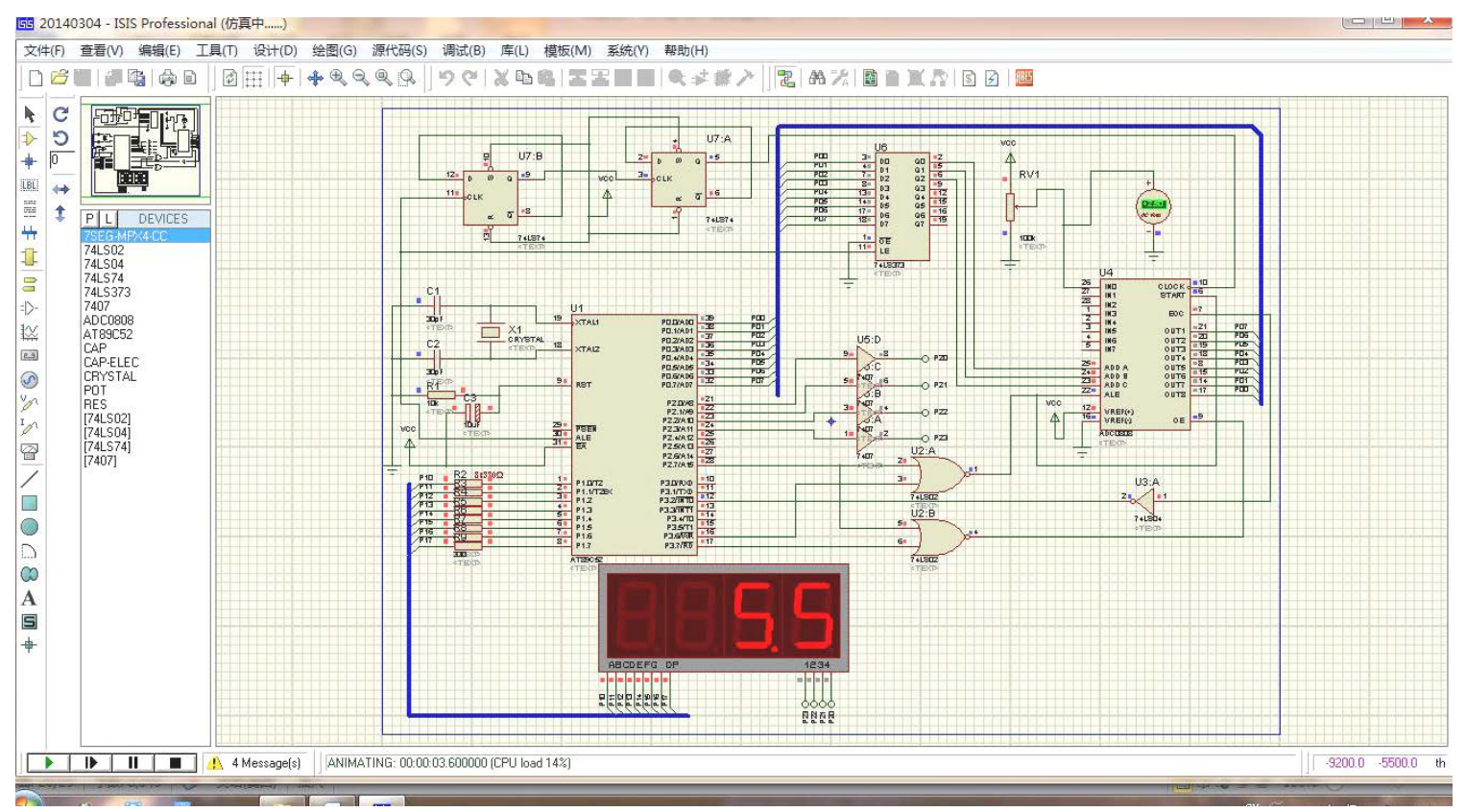

Fig.2 the picture of system simulation

\section{2 simulation analysis for the data acquisition module}

In the simulation, using $5.5 \mathrm{~V}$ dc voltage as input of the data acquisition module. That is to say if the data sampling module get the $5.5 \mathrm{~V}$ voltage signal, and then after the simulation and the micro-controller load the source program, the micro-controller exports the address signals to control ADC0808 chips collecting data and exporting digital display tube needed electrical signals. The digital tube accepts the output signal from the single chip microcomputer and displays it. Then the experiment simulation is success.

\section{3 problems and solutions about the system simulation}

We adopt the method of traceability to fundamentally solve the appeared problems, the main measures are as follows:

(1) to work more carefully, to ensure the schematic diagram is reasonable and clear, prevent conflict;

(2) find out the unreasonable problem and correct them;

(3) contrast the required functions and requirements, to select the device.

\section{Conclusion}

It is very important to test the dynamic performance parameters of the Servo system during the process of equipment design, development, production and acceptance process. With the continuous development of equipment and increasing demand for equipment, it puts forward higher requirements on test system. In order to meet the new requirement of dynamic parameter test about servo system, it is very necessary to study the dynamic performance test to solve the problem of parameter test synchronously.

In this article, through establishing the overall frame of the integrated test system for dynamic performance of the servo system, and designing the scheme respectively from two aspects of hardware and software to, the hardware of the data collection system is designed and realization.

\section{References}

[1] Li quan-xi. Single chip microcomputer principle and application simulation examples. Beijing university of aeronautics and astronautics press, 2012

[2] Zhu Linzhang. Testing theory and its application. Machinery industry press, 1987 
[3] Ye Yinzhong. Dynamic system fault detection and diagnosis method. The information and control, 1986

[4]Zhou dong-hua. Control theory and application of fault detection and diagnosis technology, 1991

[5] Xu Li. Vc + + programming guide. Science press, 1995 